Proc. XIX International School of Semiconducting Compounds, Jaszowiec 1990

\title{
PHYSICS OF GaAs/AlAs SUPERLATTICES
}

\author{
R. Planel and F. Mollot \\ Laboratoire de Microstructures et Microélectronique, \\ Centre National de la Recherche Scientifique, \\ 196, avenue Henri-Ravera, F-92220 Bagneux, France
}

(Received August 8, 1990)

\begin{abstract}
We describe the main problems encountered in MBE growth of GaAs/AlAs superlattices and heterostructures. Then, basic features for the understanding of their electronic properties are given, in the envelope-function formalism, and some related optical experiments are reviewed.
\end{abstract}

PACS numbers: 68.55.Bd, 78.65.Fa

\section{Introduction}

The interest in GaAs/AlAs heterostructures, and mainly superlattices, up-raised in the middle of the eighties, although a few preliminary works can be noticed before this period [1]. In that period, the potentialities of $\mathrm{GaAs} / \mathrm{Ga}_{1-x} \mathrm{Al}_{x} \mathrm{As}$ with $x \leq 0.35$ were already well established, and such a delay is not easily explained. However, with time, several causes and motivations might be found, which probably reinforced the natural progress of research.

First of all, there was the progress in epitaxial growth techniques, mainly Molecular Beam Epitaxy (MBE) and Metal Organic Chemical Vapor Deposi-' tion (MOCVD). This allowed a reasonable control of AlAs growth, which was, and remains today, more difficult to master than the one of GaAs and $x \leq 0.35$ $\mathrm{Ga}_{1-x} \mathrm{Al}_{x} \mathrm{As}$ alloys.

Second, an original idea came out in several laboratories [2], especially involved in laser research, where thick layers of high $\mathrm{Al}$ content $\mathrm{GaAlAs}$ alloys are required: it consisted in replacing the random traditional alloys by Short Period Superlattices (SPS's) GaAs/AlAs, with the same average Al concentration; these SPS's were supposed to act as "pseudo-alloys", especially from the point of view of average optical properties such as the refraction index. It often revealed to give better results. To illustrate these two "technological" features, we shall shortly describe some of the problems encountered in MBE in part 1 of this paper. 
A motivation of a different kind was rapidly found to be hopeless, as we shall see, but seems to have played some role [3]; it is related to the electronic properties of the random GaAlAs alloys with $x \geq 0.35$, which are indirect gap semiconductors and thus have poor optical properties from the point of view of emission devices: the use of SPS's could lead to a folding of the Brillouin zone, and thus make a direct gap material to increase the strength of optical transitions. One could have imagined laser emitting at higher energy than the direct-indirect crossover energy of GaAlAs alloys.

Another proposal to improve the electronic properties of GaAlAs alloys was made in the same period [4]: it was motivated by the existence of DX centers, deep donors with local relaxation effects which give rise to persistent photoconductivity; these centers appear, and seem unavoidable, in $n$-doped GaAlAs alloys with $0.25<x<0.45$, that is in the vicinity of the direct-indirect gap crossover concentration. Among several hypothesis, it was suggested that the random local environment of the donor atoms could be responsible for their properties. Thus replacing random alloys by SPS's, and doping selectively the GaAs (and/or AlAs) layers could help to get rid of DX centers. Although the first studies were claimed to be promising, the problem is still lying, but looks intricate enough to remain the subject of active research up to now.

Nevertheless, these proposals gave additional motivations to the study of electronic properties of SPS's. The most interesting question which arose, was the following: how to describe the crossover between direct and indirect gap behavior, which should appear in the limit of very short period and very high $\mathrm{Al}$ content. It turned out that "indirect" SPS actually belongs to "superlattice type II", that is fundamental conduction and valence states are not preferentially localized in the same layers. These studies are reviewed in parts 2 and 3 of this paper.

\section{MBE growth of GaAs/AlAs heterostructures}

The description of MBE may be found in numerous review papers [5]. Let us remind the main features we need for our purpose, which are to describe the specific interest of GaAs/AlAs heterostructures, and the problems encountered. The substrate temperature must be high enough to avoid the arsenic condensation, and low enough for the compound re-evaporation to be negligible. Within this range, typically $200^{\circ} \mathrm{C}$ to $700^{\circ} \mathrm{C}$ for GaAs, the stoichiometry is insured if the arsenic flux is in excess (with respect to the group III atom flux and to the arsenic re-evaporation), and growth rates may be controlled easily by the group III atom fluxes. However, the good quality of MBE grown structures, and the numerous new quantum effects which have been evidenced in these structures, rely on the fact that the actual growth is as close as possible to the idealistic monolayer-by-monolayer model, which would provide perfect interfaces, at the atomic scale. This is the "2D growth", the realism of which is not so easy to prove experimentally. As a matter of fact, various experimental accesses to the interface roughness have been proposed and used, for example, high resolution electron microscopy [6], quantum well exciton luminescence line shape [7], confined optical phonon Raman scattering 
[8]... Each of these techniques looks interesting and useful, but probes the roughness with its own physical phenomenon and its own length scale, different from the others. Thus contradictory results are often obtained.

However, there is a general agreement to associate the "quality" of interfaces to the mobility of group III atoms on the growing surface, as may be easily understood: atoms have a better chance to find the most appropriate location, that is to complete the growing atomic layer before an additional one is initiated. The main parameter acting on surface mobility is the growth temperature $T_{G}$, as may be easily understood. It was proved experimentally, and quantitatively measured, observing the time oscillations of intensity of Razing High Energy Electron Diffraction (RHEED) [9]. On the other hand, the too high $T_{G}$ must be avoided: it favours exchange between the different group III atomic species (e.g. $\mathrm{Ga}$ and $\mathrm{Al}$ ), leading to graduate interfaces, and also selective desorption of $\mathrm{Ga}$ with respect to $\mathrm{Al}$, which alters the control of the desired structure parameters (layer thickness, alloy concentration). Actually, the optimum growth temperature, which may depend on other growth parameters and on the structure properties to be optimized, generally lies around the Temperature of Congruent Sublimation (TCS) of the compound.

The main problem for growing GaAs/AlAs heterostructures relies on the fact that the TCS of GaAs is about $600^{\circ} \mathrm{C}$, and the one of AlAs is around $800^{\circ} \mathrm{C}$, such a difference reflecting the stronger chemical bonding of AlAs. In other words, the mobility of $\mathrm{Al}$ atoms at a given temperature is much smaller than the one of $\mathrm{Ga}$ atoms. To solve the problem, it may be proposed to change rapidly $T_{G}$, within a few seconds, from GaAs to AlAs. This may require an adaptation of the substrate oven and of the parameters of regulation. It does not seem widely used for the growth of various short period structures. The solution adopted in our laboratory is the following: first, setting $T_{G}$ at some intermediate value, e.g. $640^{\circ} \mathrm{C}$, still reasonable for $\mathrm{GaAs}$, provided the arsenic re-evaporation (since $T_{G}>\mathrm{TCS}$ ) is compensated by an additional arsenic flux; then, acting on the other parameters to improve the mobility of $\mathrm{Al}$ atoms. These parameters are the growth rate, the background purity and the arsenic flux.

The influence of growth rate on the group III atom diffusion is easily understood: the atoms have "more time" to reach an appropriate location at slower rates. The benefit, from this point of view, of growth interruption has been evidenced [10]. For obvious practical reasons, as well as to minimize the incorporation of background impurities, there are lower limits. We found that rates of, typically, $0.7 \mu \mathrm{m} / \mathrm{h}$ realized a good compromise between structure quality and growth time.

The background level of impurities, which is not really a "parameter" which one enjoys to play with, has a more dramatic influence on the growth of Al rich compounds than for GaAs. This is commonly imputed to the chemical reactivity of $\mathrm{Al}$, for example with residual $\mathrm{CO}$ molecules, and to the segregation of these impurities at the growing surface. In the case of thick layers, the accumulation of impurity may spoil the morphology itself of the resulting structure, whereas, in the case of $\mathrm{GaAs}$, only the electronic properties would be affected.

The arsenic flux also has some influence on the surface diffusion of group III atoms. This is also evidenced by several experiments, the more quantitative being 
RHEED oscillation. Provided stoichiometry conditions remain fulfilled, the minimization of this flux improves the quality of structures in most cases, and, in the case of AlAs, smooths down surfaces and interfaces. A limiting case of optimization is provided by "Migration Enhanced Epitaxy" (MEE) [11], where the arsenic flux is interrupted during the deposition of, approximately, one monolayer of group III atom. The MEE technique is not well suited for the growth of thick structures, since it requires several shutter motions per monolayer, but it may help to improve the quality of critical interfaces. In most practical cases we use conventional MBE technique with comparable growth rates for GaAs and AlAs. But we optimize the arsenic flux for the Al-rich compounds, and use an additional arsenic cell for the growth of Ga-rich compounds the TCS of which is below $T_{G}$. The careful observation of surface reconstructions during growth, which is revealed by RHEED patterns, is actually necessary to reach the optima.

We are now in position to discuss the first "technological" interest for GaAs/ /AIAs SPS, which was evidenced in structures where thick and Al-rich layers are necessary, such as lasers and other optical devices for guided optics. Whereas the growth of $\mathrm{GaAs}$, when realized in good conditions, appears to be smoothing, tending to erase imperfections as evidenced every working day by the improving role of GaAs buffer layers, this is not always the case for Al-rich alloys. Moreover, due to the essential role of residual impurities in the roughing processes, results may lack of reproducibility. Last, the surface roughness and impurity segregation develop as the layer thickness increases; due to the smoothing tendency of GaAs, the first quantum well deposited on a $1 \mu \mathrm{m}$ thick GaAlAs layer, which should be compared to a thin homogeneous film, may look better like a collection of dirty ponds with random shapes and dimensions. On the contrary, during the growth of GaAs/AlAs SPS, the roughness and contamination developing in AlAs are expected to be smoothed in the next GaAs monolayers, and the damages are not cumulative.

This may reveal to be of special interest for structures and devices which make use of optical and electronic confinement; in these cases, should coexist layers of very different thicknesses, in the ratio of optical and electronic wavelength. As an example, we have grown specially designed structures for the study of quantum well absorption with light propagating in the plane of the layer. The $3 \mathrm{~nm}$-thick GaAs QW was imbedded in a waveguide consisting of several $0.5 \mu \mathrm{m}$ thick pseudo-alloy layers with average $\mathrm{Al}$ contents 0.9 and 0.5 . The quality of this quantum well, revealed by low temperature luminescence experiments, was quite comparable to standard results.

\section{Electronic properties: Envelop-Function Approximation theoretical results}

The electronic properties of GaAs/AlAs SPS have revealed a remarkable experimental test for the validity of the Envelope-Function Approximation (EFA), as applied to the case of heterostructures by Bastard [12], for mainly two reasons:

- because of the short length scales involved in the problem, EFA should, 
in principle, be no more valid; actually, it seems to remain in a very good agreement with experiments.

- because the "barrier" material is of indirect gap, it must be carefully applied and extended to all extremes in the Brillouin zone.

We must remind that EFA allows to operate on the slowly varying part of the electronic wave function, only if the rapidly varying part is the same, or very close to, in both materials. Thus, to play with potential (and effective mass) modulation, to solve pseudo-Schroedinger equations, is valid provided the effective potential profiles are related to band extreme with the same symmetry. Thus, we are led to consider three different potential (and effective mass) variation profiles along the growth direction for, respectively, $\Gamma, L$ and $X$ minima of the host material conduction bands; in the EFA framework, these three designs are independent from each other, since any account of mixing between wave functions of different symmetries goes beyond the EFA.

The $\Gamma$ - and $X$-related potential profiles are drawn in Fig. 1 for the case
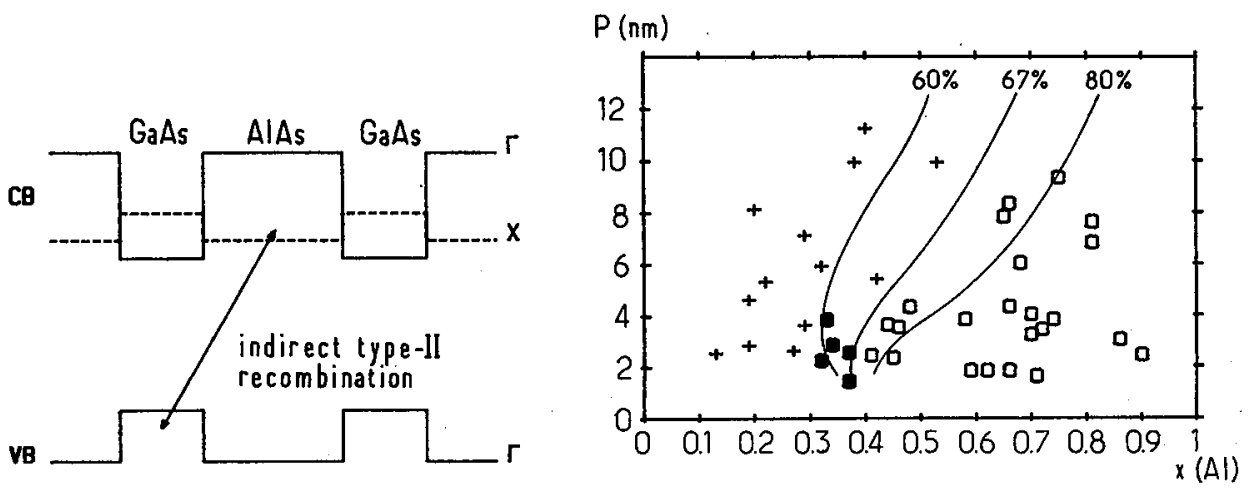

Fig.1. Potential profiles for valence, $\Gamma$-related conduction extrema (solid lines) and $X$-related conduction extrema, in a GaAs/AlAs superlattice. The gap energy is not to scale, but the offset parameter $Q_{C}=0.67$ (after [13]).

Fig. 2. Investigated GaAs/AlAs SPS are displayed on this period versus $\mathrm{Al}$ concentration map. The theoretical crossover curves between direct and "indirect" regions are shown for various values of the offset parameter $Q c$. Samples are classified as direct $(+)$ or indirect (o) on the criterion of PLE spectra; some ambiguous ones $(\bullet)$ are difficult to assign on this basis (after [13]).

of GaAs/AlAs heterostructures. (Actually, the $L$-related states reveal to lie, in these cases, at higher energies, and thus are of little interest for our purpose). The band gap energies being known otherwise, the essential parameter to position one material band energies with respect to the other is the "offset parameter", often expressed as the ratio $Q_{c}$ of the conduction offset to the gap offset, at the $\Gamma$-point. Only such a convention allows to expect this parameter to be little dependent 
on Al concentration of the alloy, even when one of the material is indirect. For $Q_{c}<0.84$, which is now well established, the $X$-profile actually belongs to type II. Since electron masses, for both host materials, are much lighter at $\Gamma$-point than at $X$-point of the Brillouin zone, one may reach the situation where the energy of $\Gamma$-states overcomes that of $X$-states, due to confinement. Then, the conduction ground state is $X$-related and is preferentially located in the AlAs slab.

This is how the direct to "indirect" crossover occurs in GaAs/AlAs SPS. The SPS theoretical parameters for this occurrence share into two regions the $P / x$ map of Fig. 2, where we have defined the SPS period: $P=L_{\mathrm{GaAs}}+L_{\mathrm{AlAs}}$, and the mean Al concentration: $x_{\mathrm{Al}}=L_{\mathrm{AlAs}} / P$. Typically, for the value $Q_{c}=0.67$ that we have determined [13], we get "indirect" type II SPS when $L_{\mathrm{GaAs}}<4 \mathrm{~nm}$ and $x_{\mathrm{Al}}>0.35$ (these values depend slightly on the bulk material parameters used, and thus on temperature). As we shall see below, these EFA predictions are surprisingly well confirmed by photoluminescence and other spectroscopic experiments made up to now.But it is still possible, and helpful, to go further into the details of the $X$-state symmetry, within this framework.

As a matter of fact, the six $X$ conduction band minima, which are situated near the Brillouin zone boundary in the wave vector directions $k \|[001]$ and equivalents, are degenerate in the bulk materials. But they are no more equivalent in our problem; the situation for minima $X_{z}$, lying along the growth direction, should be different from $X_{x}$ and $X_{y}$. In the EFA framework, it expresses through the mass anisotropy at $X$-points: $m_{L}>m_{T}$. In all cases, the relevant mass for confinement effects is $m_{z}$, which is longitudinal (i.e. $\| k$ ) in $X_{z}$, and transverse in $X_{x, y}$. Thus, the confinement energy of $X_{z}$ states should be lower. This energy difference is smaller than $100 \mathrm{meV}$ in most cases. Actually, several experimental investigations (see below) lead to the conclusion that this approach could explain their results, provided that was accounted for the initial splitting between $X_{z}$ and $X_{x, y}$ states, due to the slight lattice mismatch between AlAs and the GaAs substrate. The effects of this mismatch are generally neglected in the GaAs/AlAs system. Despite the poor knowledge of most bulk AlAs parameters, it was possible to estimate the initial splitting between 15 and $20 \mathrm{meV}$ with ground state of $X_{x, y}$ symmetry $[14,15]$. As a consequence, we may now distinguish two types of "indirect" GaAs/AlAs SPS:

- Those where the confinement energy of $X_{x, y}$ states is not sufficient to reverse this energy ordering. It remains a wave vector difference between valence and conduction fundamental states, due to $\exp \left(i k_{x} x+k_{y} y\right)$ terms in the wave functions of the latter state. These SPS are, strictly speaking, indirect gap material in the $k$-space.

- Those where this ordering reversal occurs may be named "pseudo-direct", since the envelope-function is no more of exponential form. This situation is often referred to as "folding of the Brillouin zone".

The boundary between the two situations is shown on the $L_{\mathrm{AlAs}} / L_{\mathrm{GaAs}}$ map of Fig. $3 \mathrm{~b}$, for various values of the mismatch-induced initial splitting. Roughly speaking, the real indirect situation occurs for $L_{\mathrm{AlAs}} \geq 7 \mathrm{~nm}$ or for $L_{\mathrm{GaAs}} \leq 0.8 \mathrm{~nm}$, 

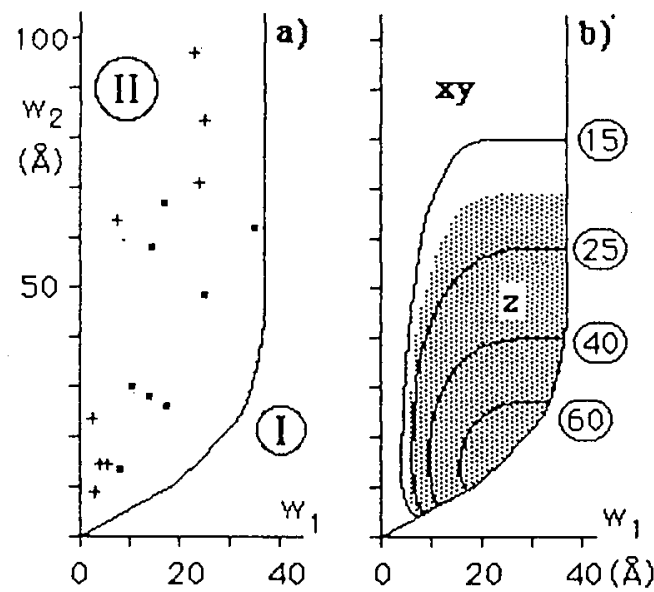

Fig. 3. a) Investigated "indirect" GaAs/AlAs SPS are displayed on this $L_{\mathrm{AlAs}}\left(w_{2}\right)$ versus $L_{\mathrm{GsAs}}\left(w_{1}\right)$ map and classified as pseudo-direct $(\bullet)$ or real indirect $(+)$ on the basis of PL spectra, as shown in Fig. 5. b) On the same map, theoretical crossover curves between the pseudo-direct $(\mathrm{z})$ and indirect (xy) regions are shown for various values of the initial mismatch-induced splitting of the $X$-states. The shaded area fits the experimental classification of a) (after [15]).

conditions to be in the type II region being otherwise fulfilled.

\section{Electronic properties: experimental studies}

We shall restrict in the following to main studies which allowed to ground the above theoretical description on experimental basis. At the present time an extensive and critical review of existing literature on the subject would oversteps the limits of this paper.

To prove the possible "indirect" (roughly speaking at the present time) character of GaAs/AlAs SPS, the basic experiment was Photoluminescence (PL), including Excitation Spectroscopy (PLE). Several groups [13, 16-19] made comparable experiments and reached about the same conclusion.

Expected type II SPS exhibit luminescence at the energy presumed for staggered luminescence, that is from AlAs confined electrons to GaAs confined holes. This PL is generally weaker than in type I SPS, which may be understood, since it is governed by the weak overlap of envelope functions. Actually, radiative lifetimes as long as hundreds of ms have been measured for this luminescence, with strong dependence on the SPS parameters and on the observed transition $[15,20,21]$.

Its energy provides a precise access to the offset parameter between GaAs and AlAs, since recombination occurs "across" the interface. Actually, in the middle of the eighties, this was still a subject of controversy, since the Dingle's rule [22] $\left(Q_{C}=0.85\right)$ had just been questioned [23]. We found $Q_{C}=0.67$, the precision 
of which is probably more limited by the poor knowledge of bulk AlAs parameters, than by the method itself. Later, photoconductivity measurements allowed to observe the same type II transitions without restriction to low temperatures, and thus to measure the (weak) temperature dependence of this offset parameter [24].

The "indirect" character was proved mainly by PLE: significative luminescence was not obtained for exciting energies lower than the expected direct, type I, and $\Gamma$-related energy gap. On this basis, SPS could be classified experimentally as shown in Fig. 2, with some uncertainties in the vicinity of the crossover parameter values. More accurate methods grounded on Optically Detected Magnetic Resonance [25] and Spin Orientation by Optical Pumping [26] have been also tested more recently.

It is worth pointing out that the $X$ - or $\Gamma$-related gap energies measured in PL or PLE experiments, performed by several groups on many various structures, where generally found to be in an excellent agreement with EFA calculations of the type described above, in both type I and type II structures. This is illustrated in Fig. 4. Although we did not make systematic studies, a long standing experience

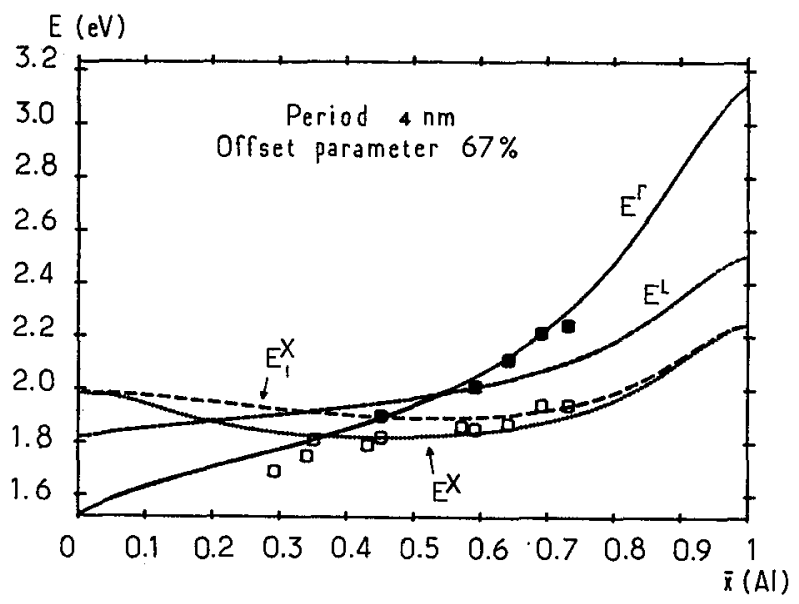

Fig. 4. Calculated energy gaps of different origins for GaAs/AlAs SPS of fixed period $4 \mathrm{~nm}$, as a function of $\mathrm{Al}$ concentration (solid lines). Experimental values are obtained from PL (o) and PLE (๑) measurements (after [13]).

allows to assert that the discrepancy between EFA calculations and experiments is generally smaller than $10 \mathrm{meV}$; provided that the SPS parameters can be measured in an independent way (most often $X$-ray diffraction), that excitonic effects are accounted for, and that parasitic effects, such as exciton localization, do not interfere.

The Type II character of $\mathrm{AlAs} / \mathrm{Ga}_{0.6} \mathrm{Al}_{0.4}$ As heterostructures was, to our knowledge, first suspected by transport measurements [27]. To prove that electrons and holes are spatially separated, electric fields have been applied, perpendicular 
to the layer $[28,29]$. Roughly speaking, it was expected that the type II transition strength and energy increase, due to the increased overlap of wave functions on both sides of one out of two interfaces, and due to the potential difference between the adjacent layers of interest. This behavior contrasts with type I systems where prevail the decrease of oscillator strength and the low energy shift due to the quantum confined Stark effect. As a consequence, in some cases, a type II to type I crossover was reached, which gave an experimental access to the $\Gamma-X$ mixing value, a quantity which is accounted for by more sophisticated calculations than the EFA theory described above. Although surprisingly, it is rather small, typically a few $\mathrm{meV}$, which confirms the advantage of EFA.

The first electric field results were promising but, to our opinion, not yet fully understood. The intensity increase is not well encountered by the calculation of envelope function overlap in the ground states; and the detail of spectra is not easy to link with the zero-field results. Perhaps the transport under electric field phenomena on the one hand, the interface roughness influence on spectra on the other hand, should be taken into better account.

The question of detailed symmetry of the conduction $X$ states which appear in type II SPS luminescence has been investigated, with the help of uniaxial strain perturbation $[14,30]$, time-resolved spectroscopy $[15,20-21]$, and optically detected magnetic resonance [25]. Although the analysis of results is intricate, the situation seems now rather clear: The two situations described above reveal at first glance in the steady-state PL spectra, provided the samples are of good quality and the exciting power is low enough to avoid high density effects (which are favoured by the very long carrier lifetimes). Two representative spectra are shown in Fig. 5, for pseudo-direct and real indirect SPS respectively. In the former case, a zero-phonon line prevails, with generally much smaller phonon satellites. In the latter case, the zero-phonon line intensity is much smaller, comparable to the phonon assisted transitions. The classification of various SPS have been done on the basis of the PL spectra and it looks in excellent agreement with the theoretical predictions, as shown in Fig. 3a. Thus, the folding of the Brillouin zone is evidenced experimentally, although the spatial separation of carriers keeps the optical transition probabilities at low values, and disappoints the dream of direct gap SPS with energy higher than the direct gap alloys.

In both cases, the results evidence heterogeneous spectral broadening, which is attributed to interface roughness as it is in the standard type I quantum well; however, the localization effects seem more important, as expected, due to the heavier mass of $X$ electrons. This localization is thought to induce mixing between $X_{z}$ and $X_{x, y}$ states.

\section{Conclusion}

We first point out the remarkable and surprising validity of EFA calculations, in both SPS types. In the type I, direct structures, it was tested for very short periods in the GaAs/AlAs system, down to 1 or $2 \mathrm{~nm}$. In the type II, "indirect" regime, the validity of EFA for $X$ states is no more questionable; moreover, if 


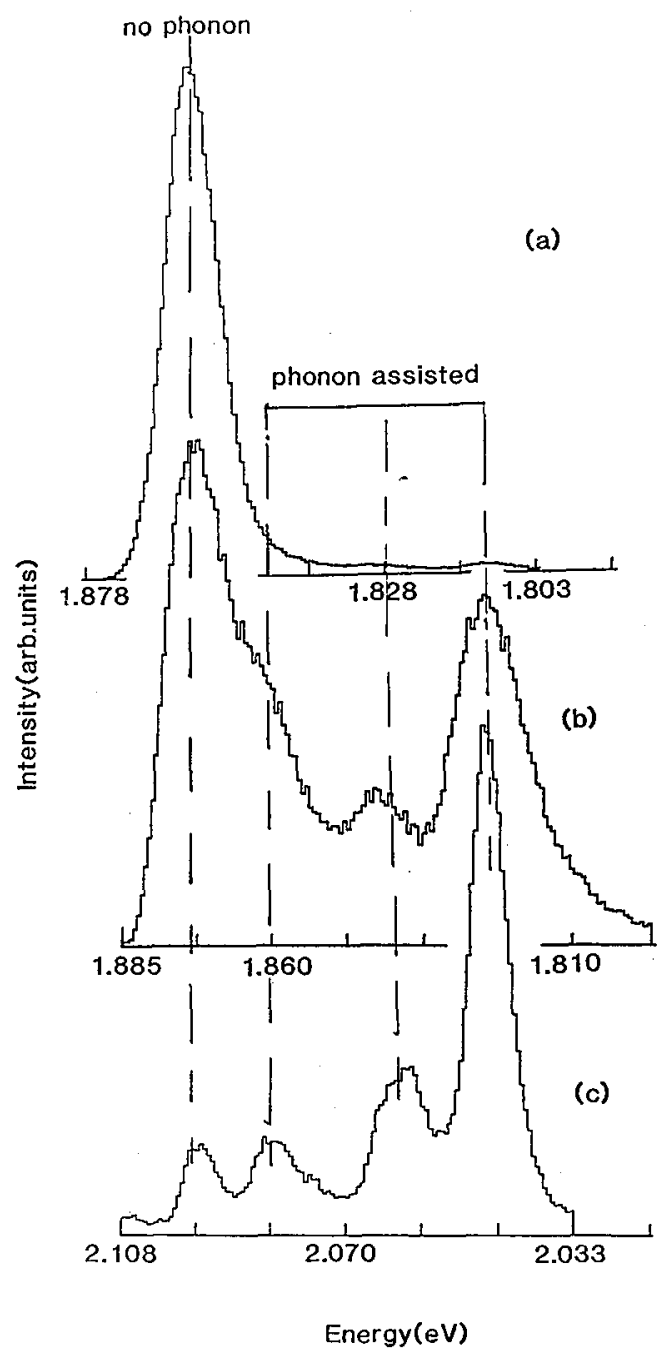

Fig.5. Representative PL spectra for three GaAs/AlAs SPS, belonging to pseudo-direct (a), and indirect (c) regions shown on Fig. 3. The SPS (b) is close to the boundary. (after [15]).

mismatch splittings are properly taken into account, it may explain the $X_{z} / X_{x, y}$ ordering with precision.

As a more prospective conclusion, we stress that the existence of type II structures in the $\mathrm{GaAs} / \mathrm{GaAlAs} / \mathrm{AlAs}$ system widens the range of possible effects 
to explore in this otherwise standard system. For example, charge separation may be used to induce photo-created electric fields, as studied recently in our laboratory on specially designed structures [31].

\section{Acknowledgements}

Among many collaborators, we wish to thank especially for their work on this subject and for numerous valuable discussion, G. Danan, M. Jeżewski, and R. Teissier.

\section{References}

[1] A.C. Gossard, P.M. Petroff, W. Weigmann, R. Dingle, A. Savage, Appl. Phys. Lett. 29, 323 (1976);

J.P. van der Ziel, A. Gossard, J. Appl. Phys. 48, 3018 (1977).

[2] K. Fujiwara, J.L. de Miguel, K. Ploog, Jpn. J. Appl. Phys. 24, L405 (1985).

[3] A. Ishibashi, Y. Mori, M. Itabashi, N. Watanabe, J. Appl. Phys. 58, 2691 (1985).

[4] T. Baba, T. Mizutani, M. Ogawa, J. Appl. Phys. 59, 526 (1986).

[5] A.Y. Cho, J. Vac. Sci. Technol. 16, 275 (1979).

[6] Y. Suzuki, H. Okamoto, J. Appl. Phys. 58, 3456 (1985);

see also: A. Ourmazd, D.W. Taylor, J.Cunningham, C.W. Tu, Phys. Rev. Lett. 62, 933 (1989).

[7] C. Weisbuch, R. Dingle, P.M. Petroff, A.C. Gossard, W. Wiegmann, Appl. Phys. Lett. 38, 840 (1981);

B. Deveaud, J.Y. Emery, A. Chomette, B. Lambert, M. Baudet Appl. Phys. Lett. 45, 1078 (1984);

for more recent studies, see also: D. Bimberg, J. Christen, T. Fukunaga, H. Nakashima, D.E. Mars, J. N. Miller, J. Vac. Sci. Technol. B 5, 1191 (1987).

[8] B. Jusserand, F. Alexandre, D. Paquet, G. Le Roux, Appl. Phys. Lett. 47, 301 (1985).

[9] J.H. Neave, P.J. Dobson, B.A. Joyce, J. Zhang, Appl. Phys. Lett. 47, 100 (1985).

[10] H. Sakaki, M. Tanaka, J. Yoshino, Jpn. J. Appl. Phys. 24, L417 (1985).

[11] Y. Horikoshi, M. Kawashima, H. Yamaguchi, Jpn. J. Appl. Phys. 25, L868 (1986); Jpn. J. Appl. Phys. 27, 169 (1988).

[12] G. Bastard, Phys. Rev. B 24, 5693 (1981).

[13] G. Danan, B. Etienne, F. Mollot, R. Planel, A.M. Jean-Louis, F. Alexandre, B. Jusserand, G. Le Roux, J.Y. Marzin, H. Savary, B. Sermage, in Proc. 18th Internat. Conf. Phys. Semicond., Stockholm 1986, ed. O. Engström World Scientific, Singapore 1987, p. 719; Phys. Rev B 35, 6207 (1987). 
[14] P. Lefebure, B. Gil, H. Mathieu, R. Planel, Phys. Rev. B 39, 5550 (1989); Phys. Rev B 40, 7802 (1989).

[15] D. Scalbert, J. Cernogora, C. Benoit a la Guillaume, M. Maaref, F.F. Charfi, R. Planel, Solid State Commun. 70, 945 (1989).

[16] L. Brey, C. Tejedor, J. L. de Miguel, F. Briones, K. Ploog, in Proc. 18th Internat. Conf. Phys. Semicond. Stockholm 1986, ed. O. Engström, World Scientific Singapore, 1987, p. 727.

[17] E. Finkman, M.D. Sturge, M.C. Tamargo, Appl. Phys. Lett. 49, 1299 (1986); J. Lumin. 40, 425 (1988).

[18] K.J. Moore, P. Dawson, C.T. Foxon, J. Phys. Colloq. 5, 525 (1987);

K.J. Moore, G. Duggan, P. Dawson, C.T. Foxon, Phys. Rev. B 38, 5535 (1988).

[19] J. Nagle, M. Garriga, W. Stolz, T. Isu, K. Ploog, J. Phys. Colloq. 5, 459 (1987).

[20] E. Finkman, M.D. Sturge, M.-H. Meynadier, R.E. Nahory, M.-C. Tamargo, D.M. Hwang, C.C. Chang, J. Lumin. 39, 57 (1987).

[21] B.A. Wilson, C.E. Bonner, R.C. Spitzer, R. Fischer, P. Dawson, K.J. Moore, C.T. Foxon, G.W.'t Hooft, Phys. Rev. B 40, 1825 (1989).

[22] R. Dingle, W. Wiegmann, C.H. Henry, Phys. Rev. Lett. 33, 827 (1974); C. Weisbuch, R. Dingle, A.C. Gossard, W. Wiegmann, Solid State Commun. 38, 709 (1981).

[23] R.C. Miller, D.A. Kleinman, A.C. Gossard, Phys. Rev. B 29, 7085, (1984).

[24] J. Barrau, K. Khirouni, Th. Amand, J.C. Brabant, B. Brousseau, M. Brousseau, Phi Hoa Binh, F. Mollot, R. Planel, J. Appl. Phys. 65, 3501 (1989).

[25] H.W. van Kesteren, E.C. Cosman, F.J.A.M. Greidanus, P. Dawson, K.J. Moore, C.T. Foxon, Phys. Rev. Lett. 61, 129 (1988);

H.W. van Kesteren, E.C. Cosman, P. Dawson, K.J. Moore, C.T. Foxon, Phys. Rev. B 39, 13426 (1989);

H.W. van Kesteren, E.C. Cosman, W.A.J.A. van der Poel, C.T. Foxon, Phys. Rev. B 41, 5283 (1990).

[26] F. Fabre, J. Bandet, J. Frandon, G. Bacquet, R. Taouint, R. Planel, G.Le Roux, to be published.

[27] T.J. Drummond, I.J. Fritz, Appl. Phys. Lett. 47, 284 (1985).

[28] G. Danan, F.R. Ladan, F. Mollot, R. Planel, Appl. Phys. Lett. 51, 1605 (1987).

[29] M.-H. Meynadier, R.E. Nahory, J.M. Worlock, M.C. Tamargo, J.L. de Miguel, M.D. Sturge, Phys. Rev. Lett. 60, 1338 (1988).

[30] M.S. Skolnick, G.W. Smith, I.L. Spain, C.R. Whitehouse, D.C. Herbert, D.M. Whittaker, L.J. Reed, Phys. Rev. B 39, 11191 (1989).

[31] M. Jezewski, F. Mollot, R. Planel, to be published. 